\title{
Microglial activation and tau propagate jointly across Braak stages
}

Tharick A. Pascoal MD PhD ${ }^{1,2}$, Andrea L. Benedet PhD ${ }^{1}$, Min Su Kang BSc ${ }^{1,2}$, Joseph Therriault BSc ${ }^{1}$, Mira Chamoun PhD ${ }^{1}$, Melissa Savard MSc ${ }^{1}$, Firoza Z. Lussier BSc ${ }^{1}$, Cécile Tissot $\mathrm{BSc}^{1}$, Nicholas J. Ashton $\mathrm{PhD}^{3}$, Sulantha Mathotaarachchi MSc ${ }^{1}$, Jenna Stevenson $\mathrm{MSc}^{1}$, Gassan Massarweh PhD ${ }^{2}$, Jean-Paul Soucy MD, MSc ${ }^{2}$, Kaj Blennow MD PhD ${ }^{3,4}$, Henrik Zetterberg MD PhD ${ }^{3,4,5,6}$, Serge Gauthier MD, FRCPC ${ }^{1}$, and Pedro Rosa-Neto MD, $\mathrm{PhD}^{1,2}$.

${ }^{1}$ Translational Neuroimaging Laboratory, The McGill University Research Centre for Studies in Aging, Department of Neurology and Neurosurgery, Faculty of Medicine, McGill University, Montreal, QC, Canada. ${ }^{2}$ Montreal Neurological Institute, McGill University, Montreal, QC, Canada. ${ }^{3}$ Department of Psychiatry and Neurochemistry, Institute of Neuroscience and Physiology, The Sahlgrenska Academy, University of Gothenburg, SE 43180, Gothenburg, Sweden. ${ }^{4}$ Clinical Neurochemistry Laboratory, Sahlgrenska University Hospital, Mölndal, Sweden. ${ }^{5}$ Department of Neurodegenerative Disease, UCL Institute of Neurology, London, United Kingdom. ${ }^{6}$ UK Dementia Research Institute at UCL, London, United Kingdom.

Corresponding author: Pedro Rosa-Neto, MD, PhD.

Translational Neuroimaging Laboratory

The McGill University Research Centre for Studies in Aging

Douglas Hospital, McGill University, Montreal, QC, Canada

6875 La Salle Blvd - FBC room 3149, Montreal, QC, Canada H4H 1R3

Email: pedro.rosa@mcgill.ca

Phone: (+1) 514-761-6131 (ext. 3407) 


\begin{abstract}
Compelling experimental evidence suggests that microglial activation is involved in the spread of tau tangles over the neocortex in Alzheimer's disease (AD). We tested the hypothesis that the spatial propagation of microglial activation and tau accumulation colocalize in a Braak-like pattern in the living human brain. We studied 130 individuals across the aging and AD clinical spectrum with positron emission tomography brain imaging for microglial activation ([11C]PBR28), amyloid- $\beta$ (A $\beta)$ ([18F]AZD4694) and tau ([18F]MK-6240) pathologies. We further assessed microglial triggering receptor expressed on myeloid cells 2 (TREM2) cerebrospinal fluid (CSF) concentrations and brain gene expression patterns. We found that [11C]PBR28 correlated with CSF soluble TREM2 and showed regional distribution resembling TREM2 gene expression. Network analysis revealed that microglial activation and tau correlated hierarchically with each other following Braak-like stages. Regression analysis revealed that the longitudinal tau propagation pathways depended on the baseline microglia network rather than the tau network circuits. The co-occurrence of $\mathrm{A} \beta$, tau and microglia abnormalities was the strongest predictor of cognitive impairment in our study population. Our findings support a model where an interaction between $\mathrm{A} \beta$ and activated microglia sets the pace for tau spread across Braak stages.
\end{abstract}

\title{
Introduction
}

Microglial activation is part of the repertoire of immune responses in the human brain and is a key element associated with the development of Alzheimer's disease (AD) ${ }^{1-3}$. Microglia coexist in the brain with the hallmark pathological features of AD, amyloid- $\beta$ (A $\beta$ ) plaques and tau neurofibrillary tangles ${ }^{4,5}$. Rather than merely being an inflammatory epiphenomenon, recent studies demonstrate that microglial activation drives tau pathology ${ }^{2,6-8}$. These animal studies predict that microglial activation may be a key player in the advance of tau pathology in the living human brain leading to dementia. 
Postmortem studies suggest that the accumulation of tau tangles in AD patients follows a stereotypical pattern known as Braak stages ${ }^{9-12}$. The deposition of tau tangles in early Braak stages in the mesial temporal cortex is a typical finding in cognitively healthy populations ${ }^{9-12}$, whereas the presence of neocortical tau is normally associated with cognitive impairment. In fact, the propagation of tau over the neocortex has been postulated as the culprit of dementia symptoms ${ }^{13}$. Although it has been proposed that $\mathrm{A} \beta$ triggers the spread of tau from transentorhinal/entorhinal structures to the neocortex ${ }^{14}$, the low concentrations of $A \beta$ in mesial temporal regions challenge this framework 9,12,15,16. Thus, understanding the mechanisms by which tau tangles spatially spread, leading to dementia, is still a pressing issue in AD.

Although animal studies support the role of microglial activation in the development of tau pathology and human studies suggest that tau progresses in Braak stages, it is unknown whether microglia play a role in the stereotyped spread of tau in AD patients. Applying recent advances in brain molecular networks ${ }^{17}$, using a large group of genetically screened individuals to optimize the signal of microglia activation brain imaging ${ }^{18,19}$, and a novel tau imaging agent capable of capturing early and late Braak stages with high sensitivity ${ }^{20,21}$, we studied the association between microglial activation and tau propagation. Specifically, we used positron emission tomography (PET) imaging agents selective for $\mathrm{A} \beta$ aggregates, tau tangles, and microglial activation, as well as postmortem triggering receptor expressed on myeloid cells 2 (TREM2) gene expression brain maps and cerebrospinal fluid (CSF) concentrations to test whether signatures of ADrelated microglial activation are associated with the stages of tau spread. Inspired by experimental literature showing that microglial activation precedes tau pathology in a similarly topographical fashion ${ }^{4,5,7,22-25}$, we hypothesized that microglial activation sets the stage for tau propagation in a Braak-like pattern in AD. The characterization of microglial activation as the basis of tau spread in Braak stages would provide critical understanding of an elementary mechanism associated with the development of AD dementia and could lead to novel therapeutic strategies aiming to contain disease progression. 


\section{Materials and methods}

\section{Participants}

The study participants were enrolled in the Translational Biomarkers of Aging and Dementia (TRIAD) study, McGill University, Canada, and genotyped for the Ala147Thr polymorphism of the TSPO gene (rs6971, https://www.ncbi.nlm.nih.gov/snp/rs6971), which is a predictor of the binding affinity of the tracer $\left[{ }^{11} \mathrm{C}\right] \mathrm{PBR} 28$ to the $18-\mathrm{kDa}$ translocator protein $(T S P O){ }^{18}$. TSPO is overexpressed in the outer membrane of the mitochondria in activated microglia ${ }^{26,27}$. Based on this genotype, individuals can either be high-, mixed-, or low-affinity binders. Since $\left[{ }^{11} \mathrm{C}\right] \mathrm{PBR} 28$ signal in low-affinity binders is negligible, and mixed and high-affinity binders show inherently different signals, we used only high-affinity binders to increase the reliability of the results. All participants had clinical and cognitive assessments, including Clinical Dementia Rating (CDR) and Mini-Mental State Examination (MMSE). Cognitively unimpaired (CU) individuals had no objective cognitive impairment and a CDR score of 0. Mild cognitive impairment (MCI) subjects had subjective/objective cognitive impairment, a CDR score of 0.5, and relatively preserved activities of daily living. Mild-to-moderate sporadic AD dementia met the National Institute on Aging and the Alzheimer's Association criteria for probable $\mathrm{AD}$ as determined by a physician and had a CDR score between 0.5 and 2. We excluded participants with inadequately treated systemic conditions, active substance abuse, recent head trauma, major surgery, or presenting with magnetic resonance imaging (MRI) / PET safety contraindications. The study was approved by the Montreal Neurological Instituted PET Working Committee and the Douglas Mental Health University Institute Research Ethics Board, and written informed consent was obtained from all participants.

\section{Brain imaging methodology}

Study participants underwent a 3D MRI (Siemens) anatomical (T1) study, as well as A $\beta$ $\left[{ }^{18} \mathrm{~F}\right]$ AZD4694, tau $\left[{ }^{18} \mathrm{~F}\right] \mathrm{MK}-6240$, and microglia activation TSPO $\left[{ }^{11} \mathrm{C}\right] \mathrm{PBR} 28$ PET imaging in the same brain-dedicated scanner (Siemens High Resolution Research Tomograph). $\left[{ }^{18} \mathrm{~F}\right] \mathrm{AZD} 4694$ images were acquired at 40-70 min after the intravenous bolus injection of the tracer and reconstructed with an OSEM algorithm on a 4D volume 
with 3 frames $(3 \times 600 s){ }^{28} \cdot\left[{ }^{18} \mathrm{~F}\right] \mathrm{MK}-6240$ images were acquired at 90-110 min after the intravenous bolus injection of the tracer and reconstructed using the same OSEM algorithm on a 4D volume with 4 frames $(4 \times 300 s){ }^{21}$. Finally, $\left[{ }^{18}\right.$ F]PBR28 images were acquired at 60-90 min after the intravenous bolus injection of the tracer and reconstructed using the OSEM algorithm on a 4D volume with 6 frames $(6 \times 300 s)^{29}$. A 6-min transmission scan with a rotating ${ }^{137} \mathrm{Cs}$ point source was performed at the end of each PET emission acquisition for attenuation correction. PET images were also corrected for motion, dead time, decay, and scattered and random coincidences. In brief, PET images were automatically registered to the native T1-weighted MRI, and MRIs were linearly and non-linearly registered to the MNI space ${ }^{30}$. Then, PET images were registered to the MNI space using transformations from the PET to native MRI and from the native MRI to the MNI space. $\left[{ }^{18} \mathrm{~F}\right] \mathrm{MK}-6240$ Standardized Uptake Value Ratios (SUVRs) used the inferior cerebellum gray matter, whereas $\left[{ }^{18} \mathrm{~F}\right]$ AZD4694 SUVRs and $\left[{ }^{18} \mathrm{~F}\right] \mathrm{PBR} 28$ SUVRs used the whole cerebellum gray matter as the reference region ${ }^{21,28,29}$. Previous studies suggested that simplified methods using the cerebellum as reference offer reliable estimates for $\left[{ }^{18} \mathrm{~F}\right] \mathrm{PBR} 28$ binding, in fact with a possibly higher sensitivity than with absolute quantitation ${ }^{29}$. Finally, PET images were spatially smoothed to an 8-mm fullwidth at half maximum resolution. Abnormal PET uptake of tracers (positivity) was defined as SUVR values 2.5 standard deviations (SD) above the mean SUVR of CU young individuals, similar to what has been proposed in previous publications ${ }^{20,31,32}$. The Desikan-Killiany-Tourville atlas was used to define the regions-of-interest ${ }^{33}$. The transentorhinal cortex was defined based on an established segmentation procedure on a $1 \mathrm{~mm}$ isotropic voxel matrix in stereotaxic space ${ }^{20,34,35}$. PET Braak-like cortical stages were determined based on results from prior autopsy studies 9-12: Braak I (transentorhinal), Braak II (entorhinal and hippocampus), Braak III (amygdala, parahippocampal gyrus, fusiform gyrus, lingual gyrus), Braak IV (insula, inferior temporal, lateral temporal, posterior cingulate, and inferior parietal), Braak V (orbitofrontal, superior temporal, inferior frontal, cuneus, anterior cingulate, supramarginal gyrus, lateral occipital, precuneus, superior parietal, superior frontal, rostro medial frontal), and Braak VI (paracentral, postcentral, precentral, and pericalcarine). A TREM2 gene expression distribution image was derived from microarray data obtained 
from the open-source Allen Human Brain Atlas (www.brain-map.org) ${ }^{36}$, which is composed of mRNA expression intensity values measured on 3702 samples from 6 healthy human brains (4 males, mean age $=42.5(13.4)$, postmortem delay= 20.6 (7) hours). TREM2 mRNA expression was derived from a Gaussian process ${ }^{37}$, and downloaded from www.meduniwien.ac.at/neuroimaging/mRNA.html.

\section{Cerebrospinal fluid measurements}

CSF soluble TREM2 (sTREM2) concentration was measured using a Meso-Scale Discovery assay, as previously described ${ }^{38}$. Additionally, CSF samples were analyzed using a multiplex immunoassay analysis (Inflammation panel; Olink, Uppsala, Sweden) (https://www.olink.com/products/inflammation/) for 92 inflammation-related markers ${ }^{39}$. The list of the 92 proteins may be found in the Supplementary Table 1.

\section{Statistical methods}

Statistical models were generated using the $\mathrm{R}$ statistical software version 3.1.2 (http://www.r-project.org/); voxel-wise statistics were carried out with MATLAB software version 9.2 (http://www.mathworks.com) and VoxelStats package ${ }^{40}$. Voxelwise receiver operating characteristic curves contrasting groups provided the area under the curve for disease diagnosis. The linear associations between biomarkers were tested using Pearson correlations. Voxel-wise associations between biomarkers were tested using linear regressions accounting for age, gender, education, and $A P O E \& 4$ status. Network analysis for tau tangles $\left(\left[{ }^{18} \mathrm{~F}\right] \mathrm{MK}-6240\right)$ and microglia $\left(\left[{ }^{18} \mathrm{~F}\right] \mathrm{PBR} 28\right)$ was performed using uptake values from composite brain regions corresponding to Braak histopathological stages (Braak I-VI), derived from regions-of-interest in the DesikanKilliany-Tourville atlas ${ }^{33}$. The edge values used as the matrix elements were equated to the correlation coefficients between regions-of-interest. The interregional correlation coefficients were calculated using Pearson partial correlation accounting for age, gender, education, $A P O E \& 4$ status, and remaining Braak regions. We verified the adequacy of these associations by verifying that the residuals of the same aforementioned associations tested using linear regressions had a normal distribution, as previously described ${ }^{17}$. Networks were evaluated with a symmetric matrix, showing the strength of the 
correlations between regions. Tau and microglia matrices elements were further correlated with each other using Pearson's correlation. Matrices correlations were corrected for multiple comparisons using Bonferroni at $P<0.05$. A voxel-wise interaction model was built to test the interactive and main effects of $A \beta$ and microglia on tau pathology. Statistical parametric maps were corrected for multiple comparisons using a fast discovery rate threshold of $P<0.05$. To asses individuals' percentage of abnormal voxels, we created a voxel-wise frequency of tau positivity maps. First, we built z-score parametric maps for each subject, anchored on the normative data of CU young. Then, we generated binary maps of tau positivity for each individual as $2.5 \mathrm{SD}$ higher than the mean SUVR of CU young and averaged these maps. Finally, we calculated the percentage of abnormal voxels for each subject.

\section{Data availability}

The data presented in this study are available from the corresponding author on a request. The data are not publicly available due to their containing information that could compromise the privacy of research participants.

\section{Results}

We genotyped 503 individuals for TSPO polymorphism (rs6971). Two hundred and sixty three (52.4\%) were identified as TSPO high-affinity binders. Following the inclusion/exclusion criteria, we studied 130 TSPO high-affinity binder individuals across the aging and AD clinical spectrum (22 CU young, $64 \mathrm{CU}$ elderly, $28 \mathrm{MCI}, 16 \mathrm{AD}$ ) who had complete cognition, MRI, and PET data (Fig. 1). Table 1 shows the demographic characteristics of the population.

\section{$\left[{ }^{11} \mathrm{C}\right]$ PBR28 as a proxy of microglial activation in $\mathrm{AD}$}

We found that in vivo regional mean $\left[{ }^{11}\right.$ C]PBR28 SUVR was correlated with the postmortem regional mean TREM2 mRNA expression obtained from the Allen Human Brain Atlas in the respective brain regions (Fig. 2), indicating that microglia activation TREM2 gene expression was partially colocalized with in vivo $\left[{ }^{11} \mathrm{C}\right] \mathrm{PBR} 28$ SUVR uptake in the human brain. In addition, we found that $\left[{ }^{11} \mathrm{C}\right] \mathrm{PBR} 28$ SUVR correlated with CSF 
sTREM2 concentration (Fig. 3A). We also found that CSF sTREM2 concentration was more highly associated with $\left[{ }^{11} \mathrm{C}\right] \mathrm{PBR} 28$ uptake, tau pathology, and other AD-related processes than most of other 92 inflammation-related proteins tested (Fig. 3B, C), supporting a stronger link of our results with TREM2 microglial activation compared to other inflammation pathways (see Supplementary Table 1 and Fig. 1).

\section{[11C]PBR28 microglial activation and AD pathophysiology}

$\left[{ }^{11} \mathrm{C}\right] \mathrm{PBR} 28 \mathrm{SUVR}$ was progressively higher from CU young to CU elderly, MCI, and $\mathrm{AD}$ dementia individuals in posterior cingulate/precuneus, inferior parietal, and lateral temporal cortices (Supplementary Fig. 2). Voxel-wise receiver operating characteristic curves revealed that $\left[{ }^{11} \mathrm{C}\right] \mathrm{PBR} 28$ uptake differentiated $\mathrm{CU}$ young from CU elderly with the highest accuracy in the posterior cingulate, precuneus, lateral temporal, and inferior parietal cortices. $\left[{ }^{11} \mathrm{C}\right] \mathrm{PBR} 28$ uptake differentiate CU elderly from MCI and MCI from $\mathrm{AD}$ dementia with the highest accuracy in posterior cingulate/precuneus, inferior parietal, and occipital cortices (Supplementary Fig. 3).

Microglial activation measured with $\left[{ }^{18} \mathrm{~F}\right] \mathrm{PBR} 28$ SUVR and CSF sTREM2 correlated with elevated brain levels of $\mathrm{A} \beta$ (CSF A $\beta 42 / 40$ ratio and $\left.\left[{ }^{18} \mathrm{~F}\right] \mathrm{AZD} 4694\right)$ and tau (CSF p-tau181 and $\left[{ }^{18}\right.$ F]MK-6240) pathologies (Fig. 4A-D). CSF sTREM2 levels in CU and MCI were associated with $\left[{ }^{18} \mathrm{~F}\right] \mathrm{MK}-6240$ tau load in early Braak regions (transentorhinal, entorhinal, and hippocampus cortices) and later Braak regions, respectively, suggesting a role for TREM2 microglial activation in the spread of tau across the disease spectrum (Fig. 4E, F). The prevalence of individuals with abnormal $\left[{ }^{11} \mathrm{C}\right] \mathrm{PBR} 28$ was higher in $\mathrm{A} \beta$ positive than A $\beta$-negative subjects, and progressively higher according to the individuals' tau Braak stage (Supplementary Fig. 4).

\section{Microglia and tau networks are closely linked with one another}

Partial correlation matrices revealed that microglia levels in regions comprising Braak histopathological stages hierarchically correlated with each other from Braak I to Braak VI. Similarly, partial correlation matrices revealed that tau tangle levels correlated with each other following the hierarchical stages proposed by Braak (Fig. 5A, B). The 
elements of the microglia-microglia and tau-tau matrices were highly correlated with one another $(r=0.8574, P<0.0001)$, supporting a strong link between the patterns of microglial activation and tau accumulation in the human brain (Fig. 5C). Notably, we found that $\left[{ }^{11} \mathrm{C}\right]$ PBR28 SUVR values in the transentorhinal cortex (Braak I) independently predicted short term 1-year tau propagation over regions comprising Braak II-VI accounting for age, sex, $A P O E \& 4$ carrier status, and A $\beta$ (Fig. 5D). $\left[{ }^{11} \mathrm{C}\right] \mathrm{PBR} 28$ SUVR in other brain regions was not associated with tau propagation over 1 year.

\section{$A \beta$ potentiates the effect of microglia on tau spreading and dementia}

Interaction models showed that high levels of global $A \beta$ burden and high levels of microglial activation in the transentorhinal cortex synergistically associated with tau load predominantly confined to allocortical regions (Fig. 6A). Similarly, high levels of global $\mathrm{A} \beta$ burden and microglial activation in neocortical regions comprising Braak IV synergistically determine neocortical tau (Fig. 6B), supporting the effect of $A \beta$ in the hierarchical propagation of tau. Also, we found a synergistic interaction between global $\mathrm{A} \beta$ deposition and microglial activation on the topographical spread of tau pathology (percentage of abnormal voxels for tau pathology in the brain) (Fig. 6C). Together, these models suggested that high levels of both $A \beta$ and microglial activation are required to potentiate tau spread.

Also, we found that a synergistic interaction between $\mathrm{A} \beta$, tau, and microglia activation PET concentrations rather than their independent or grouped by two effects was highly associated with a worse MMSE score $(P=0.0003)$. Analysis of variance strongly supported that the model with the triple interaction best described this relationship than the possible reduced models $(P<0.0001)$. In addition, we found a progressive increase in the percentage of individuals with the combination of abnormal $A \beta(A)$, tau (T), and microglial activation (MA) from CU young (0\%), to CU elderly (0\%), MCI (27\%), and AD dementia (67\%). On the other hand, the prevalence of microglia activation abnormality alone or together with only $\mathrm{A} \beta$ or tau abnormalities were not different between clinical groups, suggesting that the presence of both $A \beta$ and tau is necessary for microglial activation associates with cognitive impairment (Fig. 6D). The concomitant 
presence of A/T/MA was the factor most highly associated with cognitive impairment in our population (Fig. 6E).

\section{Discussion}

Our results suggest that microglial activation and tau accumulation spatially spread in parallel following brain circuits predicted by postmortem series from the transentorhinal/entorhinal to sensorimotor cortices. In addition, our results support that $\mathrm{A} \beta$ potentiates the effect of microglial activation on tau spread. We also found evidence suggesting that when these three pathologies are present in the human brain concomitantly, they interact with each other to determine dementia.

The spatial distribution of microglial activation and tau accumulation followed a similar patterns of progression, which were previously described for tau pathology in postmortem series ${ }^{9-12}$. These findings support established in vitro literature suggesting that microglial activation occurs in a similarly stereotyped fashion as tau pathology ${ }^{7,22-25}$. Although it has been demonstrated that microglia are able to degrade tau ${ }^{41}$, one may speculate that the mechanism by which this joint propagation occurs rests on the fact that microglia may phagocytize and release exosomes containing inefficiently degraded tau, which would contaminate surrounding cells with tau pathology ${ }^{42-45}$. Indeed, recent studies confirm that microglia release tau seeds, which is the tau conformation capable of inducing tau aggregation in affected cells ${ }^{42-46}$, as well as increased levels of exosomes containing tau in $\mathrm{AD} 47,48$. Expanding upon these in vitro studies, we showed here that this join propagation of microglia and tau hierarchically follows Braak-like stages in the human brain. Our results bring together two important concepts in AD, Braak-like tau propagation ${ }^{9-12}$ and the emerging concept of the effects of microglial activation on tau spread $^{2}$. Understanding the relationship between microglia and the spatial spread of tau may prove crucial to designing new therapies targeting the interplay between these pathologies.

Although there is compelling evidence supporting that $A \beta$ is associated with neocortical tau ${ }^{14}$, the mechanisms by which $A \beta$ triggers tau spread is poorly understood. Indeed, the 
lack of a strong correlation between $\mathrm{A} \beta$ plaques and tau and the relatively low concentrations of $\mathrm{A} \beta$ in the allocortex contest a direct link between $\mathrm{A} \beta$ and tau spread from allocortical to neocortical regions $9,12,15,16$. Here, we found that $A \beta$ pathology leads to tau spread by potentiating the effects of microglial activation on tau. Our interaction model suggested that $A \beta$ pathology in the absence of activated microglia was associated with modest levels of neocortical tau. These results are in line with recent experimental literature, supporting that $A \beta$ potentiates tau propagation in the presence of activated microglia ${ }^{2}$. Together, our findings support the notion that microglial activation links the deleterious effects of $\mathrm{A} \beta$ on tau aggregation. Interestingly, similar to previous postmortem literature ${ }^{49}$, we also showed neocortical tau in the absence of activated microglia in a small number of AD subjects, suggesting that other pathways may also potentiate tau propagation.

We found that $\left[{ }^{11} \mathrm{C}\right] \mathrm{PBR} 28$ uptake was closely related to the microglial activation marker TREM2 ${ }^{50}$. In our analyses, in vivo $\left[{ }^{11} \mathrm{C}\right] \mathrm{PBR} 28$ SUVR correlated with CSF sTREM2 concentration, and it at least partially overlapped with the distribution of TREM2 gene expression obtained from the Allen Human Brain Atlas ${ }^{36}$. These results offer further evidence support $\left[{ }^{11} \mathrm{C}\right] \mathrm{PBR} 28$ as a proxy of activated microglia in the brain, and suggest that $\left[{ }^{11} \mathrm{C}\right] \mathrm{PBR} 28$ uptake may be closed related to TREM2 microglial activation pathways in $\mathrm{AD}{ }^{50}$. In fact, several previous publications suggest a link between the microglial marker TREM2 and AD ${ }^{50}$. sTREM2 has already been shown to have a positive correlation with tau biomarkers ${ }^{51}$. Interestingly, silencing TREM2 in a model presenting tau pathology in the absence of $A \beta$ exacerbates tau pathology ${ }^{52}$, which supports the idea that $A \beta$ is crucial for the deleterious association between microglia and tau. We also measured other 92 inflammation-related proteins in CSF, under the assumptions that microglial activation sTREM2 better represent our imaging results than other inflammation pathways. We found that sTREM2 was among the inflammation proteins most strongly associated with $\left[{ }^{18} \mathrm{~F}\right] \mathrm{PBR} 28$ uptake in key $\mathrm{AD}$ regions, cognitive impairment, hippocampal atrophy, tau pathology, and white matter disease. On the other hand, several other proteins showed a stronger association with A $\beta$ than sTREM2. Together, these results support a link of our $\left[{ }^{18} \mathrm{~F}\right] \mathrm{PBR} 28$ results with TREM2 microglial 
activation pathways and the notion that $\mathrm{A} \beta$ and microglial activation may be two partially independent processes that, when converge, synergistically potentiate tau pathology.

The co-occurrence of $\mathrm{A} \beta$, tau, and microglia activation abnormalities was synergistically associated with cognitive impairment and dementia. These results support models suggesting a double hit of $A \beta$ on $A D$ progression, first potentiating the effect of microglial activation on tau accumulation and then interacting with these pathologies to determine dementia ${ }^{17}$. Additionally, these results suggest a close link between microglial activation, AD hallmark biomarkers, and dementia, suggesting that microglial activation may be a key element associated with $\mathrm{AD}$ pathophysiology with the potential to be incorporated in the biological definitions of the disease ${ }^{53}$.

Our results may have implications for clinical trials. Since microglial activation in transentorhinal cortex was potentially involved in "unleashing" mesial temporal tau over the neocortex, we may predict that individuals with tau deposition confined to transentorhinal/entorhinal regions could benefit from preventive therapies targeting transentorhinal/entorhinal microglial activation. These trials could have advantages from using validated fluid and brain imaging markers of microglial activation to assess drugs' target engagement and efficacy. In fact, previous studies have already associated microglia dysfunction in the entorhinal cortex to AD progression ${ }^{54,55}$.

The strengths of the present study include the use of only high-affinity binders for $\left[{ }^{11} \mathrm{C}\right]$ PBR28 tracer, avoiding the need for corrections associated with mixed-affinity cases. The use of only high-affinity binders allowed to perform methods highly sensitive to noise associated with artificial uptake variation, such as network analyses. All PET scans were performed using a single high-resolution brain-dedicated camera to avoid sitespecific variabilities. This study has methodological limitations. It would be highly desirable to replicate our results in studies using long-term sequential biomarkers measurements at multiple time points to evaluate the longitudinal progression of pathological processes and better understand their temporal interrelationships. However, due to the limited availability of large-scale studies including microglia activation PET 
imaging, we will probably have to wait for the cohort to mature before being able to make such observations. Although it would be desirable to replicate our findings in larger populations, it is important to emphasize that, to the best of our knowledge, this is the largest study available using microglia activation PET imaging across a disease spectrum. $T S P O$ expression indirectly captures microglia ${ }^{56}$. Still, there is no technology available that has been better validated for assessing cerebral microglial activation than TSPO PET ligands. One challenge for TSPO and numerous other PET tracers targeting physiological processes is the lack of an appropriate reference region for measuring non-displaceable binding because no brain area is devoid of specific binding for those molecules. However, previous studies have suggested that the cerebellum may be a useful reference region that can substitute, with possible advantages, absolute $\left[{ }^{18} \mathrm{~F}\right] \mathrm{PBR} 28$ quantitation associated with complex set-ups for arterial blood sampling and HPLC measurements ${ }^{29}$. In fact, the use of a reference region as opposed to arterial sampling allows the design of large-scale studies, such as the one presented here. Finally, due to self-selection bias, our participants may not represent the general population.

To conclude, our results support that microglial activation is a key element linking the effects of $A \beta$ to tau spread and ultimately dementia.

\section{Acknowledgements}

The authors thank all participants of the study and staff of the McGill Center for studies in Aging. We thank Dean Jolly, Alexey Kostikov, Monica Samoila-Lactatus, Karen Ross, Mehdi Boudjemeline, and Sandy Li for assist in the radiochemistry production. We also thank Richard Strauss, Edith Strauss, Guylaine Gagne, Carley Mayhew, Tasha VinetCelluci, Karen Wan, Sarah Sbeiti, Meong Jin Joung, Miloudza Olmand, Rim Nazar, Hung-Hsin Hsiao, Reda Bouhachi, and Arturo Aliaga for consenting subjects and/or helping with data acquisition. We thank the Cerveau Technologies for the use of MK6240.

\section{Funding}

PR-N is supported by the Canadian Institutes of Health Research (CIHR) (MOP-11-51- 
31, PR-N), the Alzheimer's Association (NIRG-12- 92090, NIRP-12-259245, PR-N), Fonds de Recherche du Québec - Santé (FRQS; Chercheur Boursier, PR-N and 2020VICO-279314). P.R-N, SG, and TP are members of the CIHR-CCNA Canadian Consortium of Neurodegeneration in Aging. Canada Foudation for innovation. project 34874. KB is supported by the Swedish Research Council (\#2017-00915), the Alzheimer Drug Discovery Foundation (ADDF), USA (\#RDAPB-201809-2016615), the Swedish Alzheimer Foundation (\#AF-742881), Hjärnfonden, Sweden (\#FO2017-0243), the Swedish state under the agreement between the Swedish government and the County Councils, the ALF-agreement (\#ALFGBG-715986), and European Union Joint Program for Neurodegenerative Disorders (JPND2019-466-236). HZ is a Wallenberg Scholar supported by grants from the Swedish Research Council (\#2018-02532), the European Research Council (\#681712), Swedish State Support for Clinical Research (\#ALFGBG720931), the Alzheimer Drug Discovery Foundation (ADDF), USA (\#201809-2016862), and the UK Dementia Research Institute at UCL.

\section{Disclosure statement}

KB has served as a consultant, at advisory boards, or at data monitoring committees for Abcam, Axon, Biogen, Julius Clinical, Lilly, MagQu, Novartis, Roche Diagnostics, and Siemens Healthineers, and is a co-founder of Brain Biomarker Solutions in Gothenburg AB (BBS), which is a part of the GU Ventures Incubator Program (outside submitted work). HZ has served at scientific advisory boards for Denali, Roche Diagnostics, Wave, Samumed, Siemens Healthineers, Pinteon Therapeutics and $\operatorname{Cog} R x$, has given lectures in symposia sponsored by Fujirebio, Alzecure and Biogen, and is a co-founder of Brain Biomarker Solutions in Gothenburg AB (BBS), which is a part of the GU Ventures Incubator Program (outside submitted work). The other authors declare no competing financial interests.

\section{Uncategorized References}

1. Heneka, M.T., et al. Neuroinflammation in Alzheimer's disease.

The Lancet. Neurology 14, 388-405 (2015). 
2. Ising, C., et al. NLRP3 inflammasome activation drives tau pathology. Nature 575, 669-673 (2019).

3. Salter, M.W. \& Stevens, B. Microglia emerge as central players in brain disease. Nature medicine 23, 1018-1027 (2017).

4. Sheffield, L.G., Marquis, J.G. \& Berman, N.E. Regional distribution of cortical microglia parallels that of neurofibrillary tangles in Alzheimer's disease. Neuroscience letters 285, 165168 (2000).

5. Serrano-Pozo, A., et al. Reactive glia not only associates with plaques but also parallels tangles in Alzheimer's disease. Am J Pathol 179, 1373-1384 (2011).

6. Asai, H., et al. Depletion of microglia and inhibition of exosome synthesis halt tau propagation. Nature neuroscience 18, 15841593 (2015).

7. Hopp, S.C., et al. The role of microglia in processing and spreading of bioactive tau seeds in Alzheimer's disease. $J$ Neuroinflammation 15, 269 (2018).

8. Maphis, N., et al. Reactive microglia drive tau pathology and contribute to the spreading of pathological tau in the brain. Brain : a journal of neurology 138, 1738-1755 (2015).

9. Braak, H. \& Braak, E. Neuropathological stageing of Alzheimer-related changes. Acta neuropathologica 82, 239-259 (1991).

10. Braak, H., Thal, D.R., Ghebremedhin, E. \& Del Tredici, K. Stages of the pathologic process in Alzheimer disease: age categories from 1 to 100 years. Journal of neuropathology and experimental neurology 70, 960-969 (2011).

11. Braak, H., Alafuzoff, I., Arzberger, T., Kretzschmar, H. \& Del Tredici, K. Staging of Alzheimer disease-associated neurofibrillary pathology using paraffin sections and 
immunocytochemistry. Acta neuropathologica 112, 389-404 (2006).

12. Braak, H. \& Braak, E. Frequency of stages of Alzheimer-related lesions in different age categories. Neurobiology of aging 18, 351-357 (1997).

13. Nelson, P.T., et al. Correlation of Alzheimer disease neuropathologic changes with cognitive status: a review of the literature. Journal of neuropathology and experimental neurology 71, 362-381 (2012).

14. Jack, C.R., Jr., et al. Tracking pathophysiological processes in Alzheimer's disease: an updated hypothetical model of dynamic biomarkers. The Lancet. Neurology 12, 207-216 (2013).

15. Thal, D.R., Rub, U., Orantes, M. \& Braak, H. Phases of A betadeposition in the human brain and its relevance for the development of AD. Neurology 58, 1791-1800 (2002).

16. Pereira, J.B., et al. Amyloid and tau accumulate across distinct spatial networks and are differentially associated with brain connectivity. Elife 8(2019).

17. Pascoal, T.A., et al. Abeta-induced vulnerability propagates via the brain's default mode network. Nat Commun 10, 2353 (2019).

18. Owen, D.R., et al. An 18-kDa translocator protein (TSPO) polymorphism explains differences in binding affinity of the PET radioligand PBR28. Journal of cerebral blood flow and metabolism : official journal of the International Society of Cerebral Blood Flow and Metabolism 32, 1-5 (2012).

19. Bhatt, S., et al. PTSD is associated with neuroimmune suppression: evidence from PET imaging and postmortem transcriptomic studies. Nat Commun 11, 2360 (2020).

20. Pascoal, T., et al. [18F]MK-6240 PET for early and late detection of neurofibrillary tangles. Brain : a journal of neurology (Article in Press)(2020). 
21. Pascoal, T.A., et al. In vivo quantification of neurofibrillary tangles with [(18)F]MK-6240. Alzheimer's research \& therapy 10, 74 (2018).

22. Eikelenboom, P., et al. Neuroinflammation - an early event in both the history and pathogenesis of Alzheimer's disease. Neurodegener Dis 7, 38-41 (2010).

23. Jaworski, T., et al. Dendritic degeneration, neurovascular defects, and inflammation precede neuronal loss in a mouse model for tau-mediated neurodegeneration. Am J Pathol 179, 2001-2015 (2011).

24. Lopez-Gonzalez, I., et al. Neuroinflammatory signals in Alzheimer disease and APP/PS1 transgenic mice: correlations with plaques, tangles, and oligomeric species. J Neuropathol Exp Neurol 74, 319-344 (2015).

25. Yoshiyama, Y., et al. Synapse loss and microglial activation precede tangles in a P301S tauopathy mouse model. Neuron 53, 337-351 (2007).

26. Kreisl, W.C., et al. Comparison of [(11)C]-(R)-PK 11195 and [(11)C]PBR28, two radioligands for translocator protein (18 $\mathrm{kDa}$ ) in human and monkey: Implications for positron emission tomographic imaging of this inflammation biomarker. NeuroImage 49, 2924-2932 (2010).

27. Hobson, B.A., et al. TSPO PET Using [18F]PBR111 Reveals Persistent Neuroinflammation Following Acute Diisopropylfluorophosphate Intoxication in the Rat. Toxicol Sci 170, 330-344 (2019).

28. Cselenyi, Z., et al. Clinical validation of 18F-AZD4694, an amyloid-beta-specific PET radioligand. Journal of nuclear medicine : official publication, Society of Nuclear Medicine 53, 415-424 (2012). 
29. Lyoo, C.H., et al. Cerebellum Can Serve As a Pseudo-Reference Region in Alzheimer Disease to Detect Neuroinflammation Measured with PET Radioligand Binding to Translocator Protein. Journal of nuclear medicine : official publication, Society of Nuclear Medicine 56, 701-706 (2015).

30. Mazziotta, J.C., Toga, A.W., Evans, A., Fox, P. \& Lancaster, J. A probabilistic atlas of the human brain: theory and rationale for its development. The International Consortium for Brain Mapping (ICBM). Neurolmage 2, 89-101 (1995).

31. Lowe, V.J., et al. Widespread brain tau and its association with ageing, Braak stage and Alzheimer's dementia. Brain : a journal of neurology 141, 271-287 (2018).

32. Pontecorvo, M.J., et al. Relationships between flortaucipir PET tau binding and amyloid burden, clinical diagnosis, age and cognition. Brain : a journal of neurology 140, 748-763 (2017).

33. Klein, A. \& Tourville, J. 101 labeled brain images and a consistent human cortical labeling protocol. Front Neurosci 6, 171 (2012).

34. Tward, D.J., et al. Entorhinal and transentorhinal atrophy in mild cognitive impairment using longitudinal diffeomorphometry. Alzheimers Dement (Amst) 9, 41-50 (2017).

35. Kulason, S., et al. Cortical thickness atrophy in the transentorhinal cortex in mild cognitive impairment. NeuroImage. Clinical 21, 101617 (2019).

36. Hawrylycz, M.J., et al. An anatomically comprehensive atlas of the adult human brain transcriptome. Nature 489, 391-399 (2012).

37. Gryglewski, G., et al. Spatial analysis and high resolution mapping of the human whole-brain transcriptome for 
integrative analysis in neuroimaging. NeuroImage 176, 259-267 (2018).

38. Jensen, C.S., et al. Exercise as a potential modulator of inflammation in patients with Alzheimer's disease measured in cerebrospinal fluid and plasma. Experimental gerontology 121, 91-98 (2019).

39. Danielson, M., et al. Neuroinflammatory markers associate with cognitive decline after major surgery: Findings of an explorative study. Annals of neurology 87, 370-382 (2020).

40. Mathotaarachchi, S., et al. VoxelStats: A MATLAB Package for Multi-Modal Voxel-Wise Brain Image Analysis. Front Neuroinform 10, 20 (2016).

41. Kettenmann, H., Hanisch, U.K., Noda, M. \& Verkhratsky, A. Physiology of microglia. Physiol Rev 91, 461-553 (2011).

42. DeVos, S.L., et al. Tau reduction prevents neuronal loss and reverses pathological tau deposition and seeding in mice with tauopathy. Sci Transl Med 9(2017).

43. Furman, J.L. \& Diamond, M.I. FRET and Flow Cytometry Assays to Measure Proteopathic Seeding Activity in Biological Samples. Methods Mol Biol 1523, 349-359 (2017).

44. Takeda, S., et al. Seed-competent high-molecular-weight tau species accumulates in the cerebrospinal fluid of Alzheimer's disease mouse model and human patients. Ann Neurol 80, 355367 (2016).

45. Takeda, S., et al. Neuronal uptake and propagation of a rare phosphorylated high-molecular-weight tau derived from Alzheimer's disease brain. Nat Commun 6, 8490 (2015).

46. Leyns, C.E.G. \& Holtzman, D.M. Glial contributions to neurodegeneration in tauopathies. Mol Neurodegener 12, 50 (2017). 
47. Saman, S., et al. Exosome-associated tau is secreted in tauopathy models and is selectively phosphorylated in cerebrospinal fluid in early Alzheimer disease. The Journal of biological chemistry 287, 3842-3849 (2012).

48. Fiandaca, M.S., et al. Identification of preclinical Alzheimer's disease by a profile of pathogenic proteins in neurally derived blood exosomes: A case-control study. Alzheimer's \& dementia : the journal of the Alzheimer's Association 11, 600-607 e601 (2015).

49. Perez-Nievas, B.G., et al. Dissecting phenotypic traits linked to human resilience to Alzheimer's pathology. Brain : a journal of neurology 136, 2510-2526 (2013).

50. Gratuze, M., Leyns, C.E.G. \& Holtzman, D.M. New insights into the role of TREM2 in Alzheimer's disease. Mol Neurodegener 13, 66 (2018).

51. Piccio, L., et al. Cerebrospinal fluid soluble TREM2 is higher in Alzheimer disease and associated with mutation status. Acta neuropathologica 131, 925-933 (2016).

52. Jiang, T., et al. Silencing of TREM2 exacerbates tau pathology, neurodegenerative changes, and spatial learning deficits in P301S tau transgenic mice. Neurobiology of aging 36, 31763186 (2015).

53. Jack, C.R., Jr., et al. A/T/N: An unbiased descriptive classification scheme for Alzheimer disease biomarkers. Neurology 87, 539-547 (2016).

54. Grubman, A., et al. A single-cell atlas of entorhinal cortex from individuals with Alzheimer's disease reveals cell-type-specific gene expression regulation. Nature neuroscience 22, 2087-2097 (2019). 
55. Criscuolo, C., et al. Entorhinal Cortex dysfunction can be rescued by inhibition of microglial RAGE in an Alzheimer's disease mouse model. Sci Rep 7, 42370 (2017).

56. Dani, M., et al. Microglial activation correlates in vivo with both tau and amyloid in Alzheimer's disease. Brain : a journal of neurology 141, 2740-2754 (2018).

\section{Table}

Table 1. Demographics and key characteristics of the clinical population.

\begin{tabular}{|l|c|c|c|c|}
\hline Characteristic & CU Young & CU Elderly & MCI & AD dementia \\
\hline Number & 22 & 64 & 28 & 16 \\
\hline Age, years, mean (SD) & $23(2.4)$ & $72(5.5)$ & $73(8.6)$ & $70(7.7)$ \\
\hline Male, number (\%) & $8(36)$ & $14(22)$ & $17(61)$ & $6(538)$ \\
\hline Education, years, mean (SD) & $16.6(1.7)$ & $15.5(3.7)$ & $14.9(3.4)$ & $14.4(3.3)$ \\
\hline MMSE score, mean (SD) & $29.8(0.5)$ & $29.2(1)$ & $27(4)$ & $21(6.3)$ \\
\hline APOE \&4, number (\%) & $3(14)$ & $18(28)$ & $13(46)$ & $7(44)$ \\
\hline
\end{tabular}


